\title{
MiR-1256 suppresses proliferation and migration of non-small cell lung cancer via regulating TCTN1
}

\author{
WEI LIU, XIUWEI WAN, ZONGYUN MU, FEI LI, LEI WANG, JING ZHAO and XIAORI HUANG \\ Department of Respiratory Medicine, People's Hospital of Rizhao, Rizhao, Shandong 276826, P.R. China
}

Received November 30, 2017; Accepted May 15, 2018

DOI: $10.3892 / 01.2018 .8794$

\begin{abstract}
Mounting evidence has shown that miRNA expression is abnormal in various human cancers. Here, we mainly explored the biological function and the potential mechanisms of miR-1256 in non-small cell lung cancer (NSCLC). The miR-1256 mRNA expression was detected by quantitative real-time PCR and tectonic family member 1 (TCTN1) mRNA expression was detected by immunoblotting. The TCTN1 was identified to be the direct and specific target gene of miR-1256 by luciferase reporter assay. Cell proliferation was examined by methyl thiazolyl tetrazolium assay and migration was detected by transwell assay. MiR-1256 expression was downregulated in NSCLC tissues, whereas the expression of TCTN1 was upregulated, compared with normal tissues. We also found that overexpression of miR-1256 in these NSCLC cell lines inhibited cell proliferation and migration. Furthermore, TCTN1 was identified as a direct target of miR-1256 by luciferase reporter assays. Collectively, these data stated that the inhibitory effect of miR-1256 in NSCLC was realized by upregulating TCTN1, suggesting that miR-1256/TCTN1 axis may play a critical role as NSCLC therapeutic target.
\end{abstract}

\section{Introduction}

Lung cancer accounts for the majority of all cancer-related deaths worldwide (1). Non-small cell cancer (NSCLC) accounts for about 80-85\% of all types of lung cancer, and the survival rate is very low (2). Since almost all patients have relapses, it leads to the metastasis of disease and the death of patients. Therefore it is urgent to search for better ideas to treat human NSCLC.

MicroRNAs (miRNAs), an endogenous non-coding RNA, were found in eukaryotic organisms, with a length of about

Correspondence to: Dr Wei Liu, Department of Respiratory Medicine, People's Hospital of Rizhao, 126 Taian Road, Rizhao, Shandong 276826, P.R. China

E-mail: hfb339193@163.com

Key words: miR-1256, proliferation, migration, non-small cell lung cancer, tectonic family member 1
20 25 nucleotides. The abnormal expression of miRNAs was closely correlated with the tumor development, including regulating cell proliferation, migration, invasion and apoptosis (3-5). In recent years, microarray analysis or other advanced technologies have detected many miRNAs $(6,7)$, and increasing research has shown that miRNAs forge closer relationships with various cancers, including gastric cancer, osteosarcoma and colorectal cancer (8-10). MiR-1256 is located on chromosome 12 (11), a previous report has shown that miR-1256 expression was downregulated and it inhibited proliferation of prostate cancer and nasopharyngeal carcinoma (12). However, the study of miR-1256 in NSCLC cells has not been reported.

It has been shown that miRNAs can inhibit gene expression by degrading target messenger RNAs or blocking its translation by combining its 3'-untranslated region (3'-UTR), thereby participating in the development of cancers $(13,14)$. For example, miR-1256 mimic inhibited prostate cancer cell growth and invasion by regulating the PGK-1 (12). It has been proven that tectonic family member 1 (TCTN1) played an important role in cellular differentiation, apoptosis, and disease processes (15). Moreover, research has shown that TCTN1 regulated malignant cells growth of gastric cancer, prostate cancer, colorectal cancer and glioblastoma (16-19). It provides a novel therapeutic strategy for the treatment of cancers. However, the function of TCTN1 and how miR-1256 regulated tumor development in human NSCLC via targeting of TCTN1 has not been reported.

In the present study, we investigated miR-1256 and TCTN1 expression level in human NSCLC. The results showed that miR-1256 was downregulated, whereas, TCTN1 was upregulated in NSCLC, TCTN1 is characterised as a direct target of miR-1256 in NSCLC cells. Our results provided a new potential direction for NSCLC diagnosis and therapeutic interventions.

\section{Materials and methods}

Tissue collection. Normal tissues and normal lung cancer tissues were acquired from 30 paired patients after they signed informed consent. They had undergone surgery at People's Hospital of Rizhao (Rizhao, China) between May, 2011 and April, 2016. Then, we stored the samples at $-80^{\circ} \mathrm{C}$. All the experiments were approved by the Ethics Committee of People's Hospital of Rizhao (Rizhao, China). 
Cell culture and transfection. The NSCLC cell lines (A549 and SK-MES-1) were obtained from the Cell Resource Center, Institute of Biochemistry and Cell Biology at the Chinese Academy of Sciences (Shanghai, China). The two cell lines were cultured in RPMI-1640 (Gibco; Thermo Fisher Scientific, Inc., Waltham, MA, USA), supplement with $10 \%$ fetal bovine serum (FBS), penicillin $(100 \mathrm{U} / \mathrm{ml})$ and streptomycin $(100 \mathrm{U} / \mathrm{ml})$, which was incubated at $37^{\circ} \mathrm{C}$ under $5 \% \mathrm{CO}_{2}$ atmosphere. The cells were collected in logarithmic phase for transfection. They were transfected using DharmaFect Transfection Reagent (GE Healthcare Dharmacon, Inc., Lafayette, CO, USA) according to the manufacturer's instructions.

Western blot analysis. After transfection for $48 \mathrm{~h}$, RIPA lysis containing proteinase inhibitors (Beyotime Institute of Biotechnology, Haimen, China) and phenylmethanesulfonyl fluoride were used to extract total protein from the A549 and SK-MES-1 cells. The protein concentrations were tested with the BCA protein assay kit (Beyotime Institute of Biotechnology). A $5 \mathrm{X}$ loading buffer was diluted to $1 \mathrm{X}$ by adding into the supernatants containing total protein, subsequently, the samples were heated at $95^{\circ} \mathrm{C}$ for $5 \mathrm{~min}$, then cooled to room temperature and stored in a refrigerator until use. The total protein $(50 \mu \mathrm{g})$ was added into the hole of SDS-PAGE and performed electrophoresis at $60 \mathrm{~V}$ voltage until the bromophenol blue runs out of the bottom. The proteins were then transferred to nitrocellulose filter membranes, then, skim milk (5-10\%) was used to block the proteins on the membranes at room temperature for $2 \mathrm{~h}$. Firstly, the membranes were incubated with the primary antibody rabbit polyclonal to TCTN1 (cat. no. ab105381; 1:1,000; Abcam, Cambridge, MA, USA) at $4^{\circ} \mathrm{C}$ overnight, after washed with 1xTBST (pH 7.4) three times later; the secondary antibodies goat anti-rabbit IgG-HRP (cat. no. sc-2004; 1:3,000; Santa Cruz Biotechnology, Inc. Santa Cruz, CA, USA) were added and incubated at room temperature for $2 \mathrm{~h}$. GAPDH primary antibody (cat. no. 70699; 1:5,000; Abcam, Cambridge, MA, USA) was chosen as the internal reference. Protein bands were detected using chemiluminescence method (ECL; EMD Millipore, Billerica, MA, USA). GADPH served as a loading control.

$R N A$ isolation and reverse transcription-quantitative $P C R$ $(R T-q P C R)$. We used TRIzol reagent (Invitrogen; Thermo Fisher Scientific, Inc., Waltham, MA, USA) to extract total RNA from the cell lines or tissue samples. For the detection of miR-1256, U6 was used as an internal control, and cDNA was synthesized using a TaqMan microRNA reverse transcription kit (Applied Biosystems; Thermo Fisher Scientific, Inc.), and qPCR was performed using the TaqMan microRNA PCR kit (Applied Biosystems; Thermo Fisher Scientific, Inc.). For the analysis of TCTN1, GAPDH was used as an internal control, reverse transcription was conducted using the moloney murine leukemia virus reverse transcription system (Promega Corporation, Madison, WI, USA), followed by qPCR using the SYBR Premix Ex Taq kit (Takara Biotechnology Co., Ltd., Dalian, China). The sequences of the primers were as follows: miR-1256 GGCGCGATTTTAGTTTATC (forward) and TTT AATTAC CAACCGAATACG (reverse); for TCTN1: CCTTT GCGTGAATGTTGTTC (forward) and AGAGGGACTGGC TGGGTATT (reverse); for U6 CTCGCTTCGGCAGCACA (forward) and AACGCTTCACGAATTTGCGT (reverse); for
GADPH: ACAACTTTGGTATCGTGGAAGG (forward) and: GCCATCACGCCACAGTTTC (reverse). GADPH and U6 were used as internal reference. The relative expression level was calculated using the $2^{-\Delta \Delta \mathrm{Cq}}$ method (20).

\section{Methyl thiazolyl tetrazolium (MTT)}

Cell viability was detected by MTT assay. After transfection, A549 and SK-MES-1 cells were placed in 96-well plates and cultured for 1-4 days. Then, MTT solution (20 $\mu \mathrm{l})$ was added into each well to incubate for $4 \mathrm{~h}$ at $37^{\circ} \mathrm{C}$, then carefully removing the medium and adding $100 \mu \mathrm{l}$ dimethyl sulfoxide (Sigma; Merck KGaA, Darmstadt, Germany) per well to dissolve the formazan crystals, the plates were read at a wavelength of $490 \mathrm{~nm}$ to measure the absorbance of each well at 0 , 24, 48, 72 and $96 \mathrm{~h}$.

Transwell assay. Cell migration assays were performed using transwell chambers, $8 \mathrm{~mm}$ pore size culture inserts were placed into the 24-well plates to separate the top and the lower chambers, and $1 \times 10^{5}$ cells were added into the top chambers and RPMI-1640 medium with 20\% FBS and seeded to the lower chambers as an attractant, after cell incubation at $37^{\circ} \mathrm{C}$, $5 \% \mathrm{CO}_{2}$ atmosphere for $24 \mathrm{~h}$, the media was removed. Cotton swabs was used to remove the cells on the upper surface of the membrane, and $100 \%$ methanol was used to fix the cells migrated to the lower membrane and the $0.1 \%$ crystal violet was used to stain the cells. The numbers of cells were quantified by a microscope (Olympus Corporation, Tokyo, Japan).

Dual luciferase reporter assay. The recombinan pMIR-reportor luciferase vector was used for TCTN1 3'UTR luciferase assays. The wild-type or mutated miR-1256 binding site targeted TCTN1 3'UTR were cloned into the pMIR-reportor luciferase vector. Cells transfected with control mimic and miR-1256 mimic were collected using Lipofectamine ${ }^{\circledR} 2000$. The Dual Luciferase Reporter Assay System (Promega Corporation, Madison, WI, USA) was used to measure the luciferase activity after transfection for $48 \mathrm{~h}$.

Statistical analysis. All experiments were repeated in triplicate, GraphPad Prism 5.02 software (GraphPad Software, Inc., La Jolla, CA, USA) and SPSS 16.0 software (SPSS, Inc., Chicago, IL, USA) were used to perform statistical analyses, Student's t-test or post hoc test after one-way analysis of variance in SPSS were used to analyze the differences between the groups. Tukey's post hoc test was the post hoc test used following one-way analysis of variance. A $\mathrm{P}<0.05$ was considered to indicate a statistically significant difference.

\section{Results}

MiR-1256 expression is reduced in NSCLC cells. We used qRT-PCR to examine the miR-1256 levels in 30 paired of NSCLC and normal tissues to explore the role of miR-1256 in NSCLC. As seen in Fig. 1A, we found that miR-1256 levels were frequently downregulated in 22/30 (73\%) of the NSCLC tissues, and the miR-1256 average expression in tumor tissues was decreased compared with the normal tissues (Fig. 1B). We also detected miR-1256 expression in NSCLC tissues of varying stages, the result shows that, the expression of 


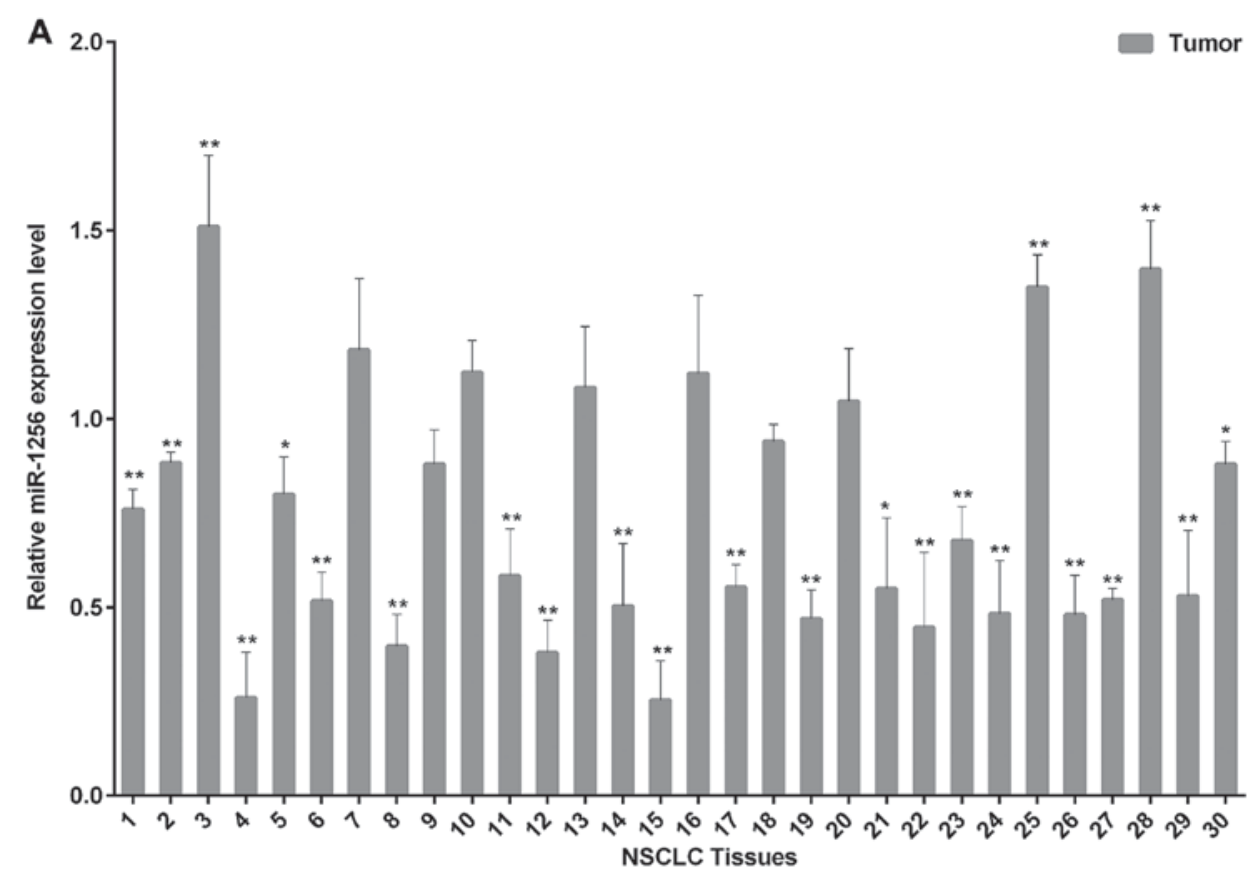

B

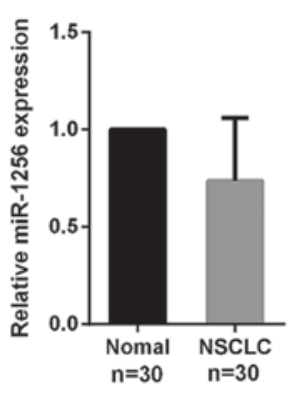

C

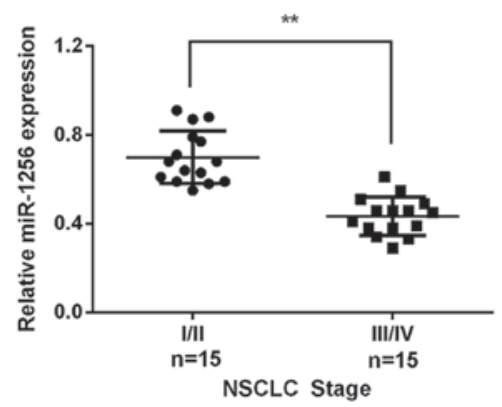

D

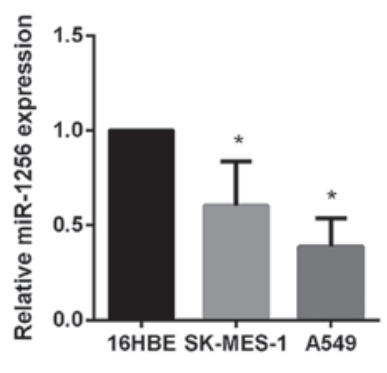

Figure 1. Decreased miR-1256 expression in NSCLC tissues and cell lines. (A) miR-1256 expression level in normal and NSCLC tissues was detected by qRT-PCR. Results for each NSCLC tissue sample were normalized to the results for their paired normal sample. (B) Quantitative analysis of the average expression of miR-1256 in NSCLC and normal tissues. (C) The miR-1256 expression level in NSCLC tissues of varying stage was detected by qRT-PCR. (D) The miR-1256 expression level detected in A549, SK-MES-1 and 16HBE cell lines. All the data are expressed as the means \pm SD. ${ }^{*} \mathrm{P}<0.05$; ${ }^{* *} \mathrm{P}<0.01$. NSCLC, non-small cell lung cancer; qRT-PCR, quantitative real-time PCR; SD, standard deviation.

miR-1256 in stages III-IV (higher stage lesions) was significantly lower than in stages I-II (lower stage lesions) (Fig. 1C). In addition, the miR-1256 expression in A549 and SK-MES-1 cell lines was also markedly downregulated, compared with normal 16HEB cells (Fig. 1D). Taken together, these data suggest that miR-1256 may play a tumor suppressive role in the progression of NSCLC.

MiR-1256 inhibited cell proliferation and migration of NSCLC. We performed an experiment to explore whether re-expression of miR-1256 could contribute to the NSCLC cell proliferation and migration. The A549 cells and SK-MES-1 cells were transfected with miR-1256 mimic or miR-1256 inhibitor, as shown in Fig. 2A and B. MTT assays was used to examine the effect of miR-1256 on cell proliferation in A549 and SK-MES-1 cells and transwell assays were used to detect the effect of the miR-1256 on cell migration in A549 and SK-MES-1 cells. As shown in Fig. 2C and D, cellular proliferation data showed that transfection with miR-1256 mimic exhibited a significant decrease to cell growth after $48 \mathrm{~h}$, while miR-1256 inhibitor increased cell proliferation remarkably in both NSCLC cell lines. The results of transwell showed that, after transfection with miR-1256 mimic, the migration ability of cells was reduced distinctly in two NSCLC cell lines and transfection with miR-1256 inhibitor, the migration ability of cells was significantly augmented (Fig. 2E and F).

MiR-1256 directly targets TCTN1. Next, we detected the underlying mechanism of miR-1256 in lung cancer cells, we used the TargetScan prediction programs (www.targetscan.org) to predict the potential targets of miR-1256, the results showed that miR-1256 may target the 3'-UTR of TCTN1 (Fig. 3A), the wild-type and mutant type of TCTN1 3'-UTR was constructed to psiCHECK-2 vector to validate whether miR-1256 regulated NSCLC cells by regulating TCTN1, then, we used luciferase reporter vectors transfected into A549 and SK-MES-1 cells to detect the luciferase activity (Fig. 3B and C), compared with the control group, miR-1256 mimic markedly decreased the relative luciferase activity in cells transfected with wild-type TCTN1 3'-UTR, but it has no significantly changed on mut type. These data proved that TCTN1 is regulated by miR-1256 in NSCLC cells. We then detected TCTN1 expression in 

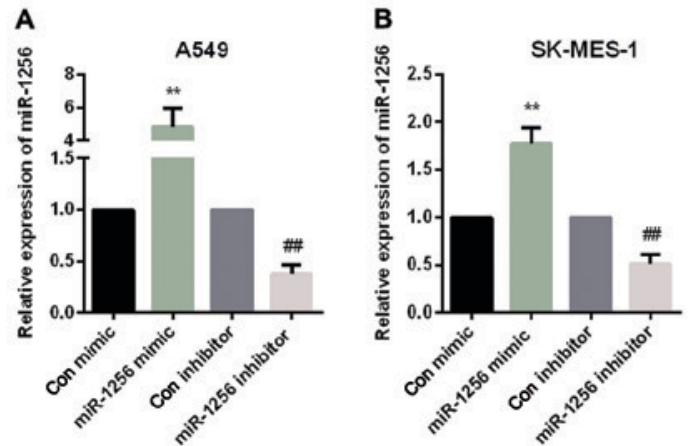

C

E

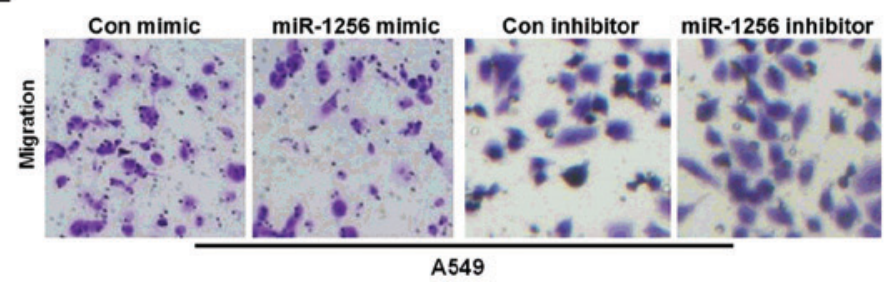

$\mathbf{F}$

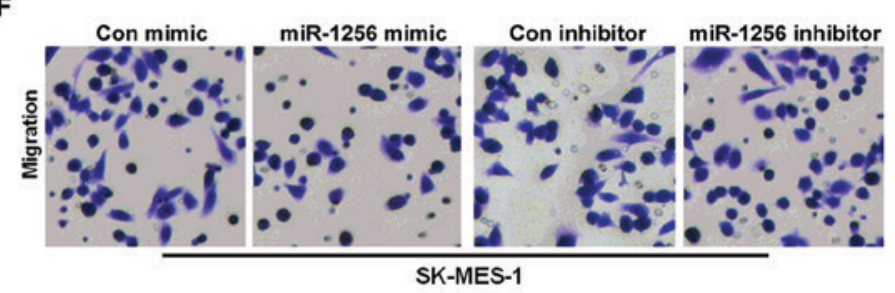

D

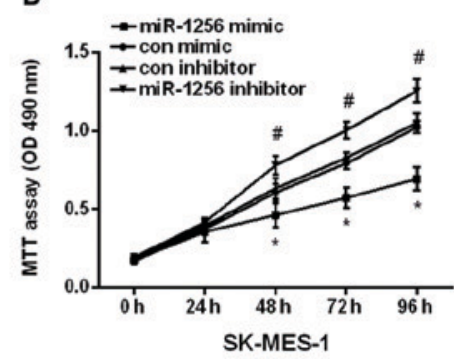

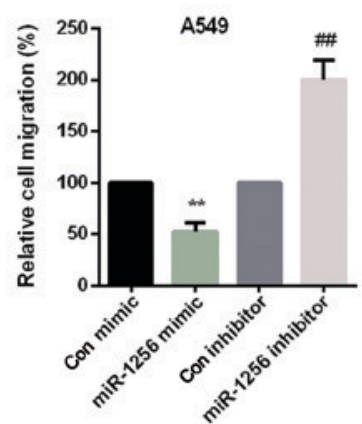

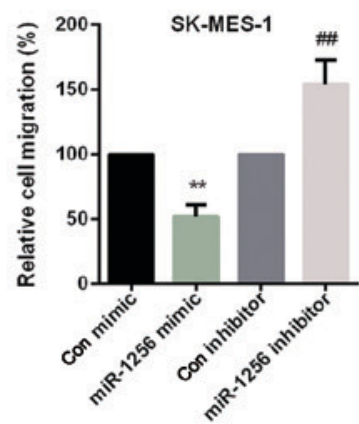

Figure 2. Inhibition of miR-1256 in NSCLC cell proliferation and migration. (A and B) MiR-1256 mRNA expression in A549 cells and SK-MES-1 cells transfected with miR-155 mimic or inhibitor was detected by qRT-PC, ${ }^{* *} \mathrm{P}<0.01 ;{ }^{\# \#} \mathrm{P}<0.01$. (C and D) MTT assay was used to detect cell proliferation after transfected with miR-1256 mimic or miR-1256 inhibitor in A549 and SK-MES-1 cells, ${ }^{*} \mathrm{P}<0.05 ;{ }^{*} \mathrm{P}<0.05$. (E and F) Transwell migration assay was used to examine cell migration after transfected with miR-1256 mimic and miR-1256 inhibitor in A549 and SK-MES-1 cell. ${ }^{* *} \mathrm{P}<0.01$; ${ }^{* \#} \mathrm{P}<0.01$. NSCLC, non-small cell lung cancer; qRT-PCR, quantitative real-time PCR; MTT, methyl thiazolyl tetrazolium assay.

NSCLC tissues and found that the expression of TCTN1 was increased (Fig. 3D). Next, we transfected two NSCLC cell lines with miR-1256 mimic or inhibitor to determine the effect of miR-1256 on the expression of TCTN1 to further confirm the above results (Fig. 3E and G), the protein level and mRNA of TCTN1 was markedly downregulated in both two NSCLC cell lines of the ectopic expression of miR-1256, while inhibiting miR-1256 significantly upregulated the protein and mRNA levels of TCTN1.

Knockdown of TCTN1 expression inhibits NSCLC cell proliferation and migration. We used TCTN1-specific siRNA to knock down TCTN1 in A549 cells to examine the potential carcinogenicity of TCTN1 in lung cancer, the transfection efficiency of TCTN1 siRNA was shown in Fig. 4A and B. MTT assay and transwell assay results showed that knockdown of TCTN1 decreased the proliferative and migratory ability of A549 cells (Fig. 4C and D), TCTN1 siRNA deprived the promoting effect of miR-1256 inhibitor on cell proliferation and migration, suggesting that TCTN1 is regulated by miR-1256 in regulating of lung cancer cells (Fig. 4E and F).

\section{Discussion}

Substantial evidence has stated that various miRNAs play critical roles in the regulating of NSCLC development by inducing mRNA degradation or inhibiting translation of a target gene (21). MiRNAs functioned as either oncogenes or suppressor genes in NSCLC (22). For example, Yang et al found that miR-126 expression level in NSCLC was downregulated and associated with poor survival as a tumor suppressor (23). However, miR-17-92 expression was increased in lung cancers, and overexpression of miR-17-92 enhanced cell proliferation (24). MiR-21 was proven to downregulate PTEN expression and to stimulate NSCLC growth and invasion (25). A previous study showed that miR-31 targeted a tumor suppressor in regulating lung cancer as an oncogene (26). A study stated that miR-1256 expression was reduced in prostate cancer (12). However, the major role of miR-1256 in NSCLC is unclear. We first examined the effect of miR-1256 in NSCLC cells to understand the role of miR-1256 in NSCLC. We found that miR-1256 was markedly downregulated, and playing a critical role in the tumorigenesis 
A Position 178-185 of TCTN1 3'UTR 5'...AGCUCCACCAGCCAUCAAUGCCA... hsa-miR-1256 3' UCGAUCACUCUUCAGUUACGGA TCTNI 3'URT Mut $\quad$ S'...AGCUCCACCAGCCAUGUAACCCA...

B

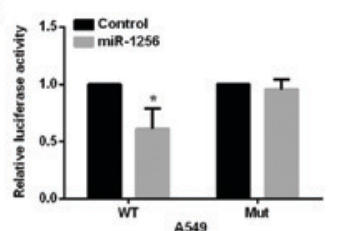

c

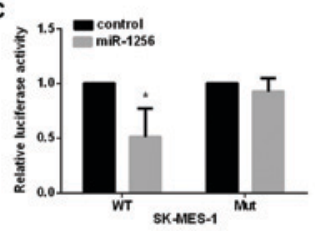

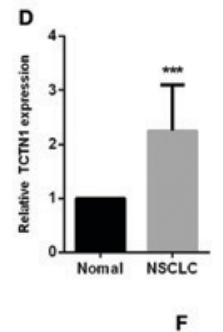

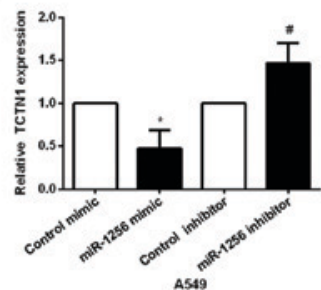

G

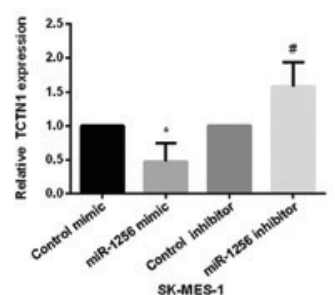

Figure 3. MiR-1256 directly targets TCTN1. (A) The schematic diagram represents the presumptive binding site of miR-1256 and the 3'-UTR of the TCTN1. (B and C) Relative luciferase activity was detected in A549 and SK-MES-1 cells after co-transfected with TCTN1 3'-UTR (wild-type or mutant) and control or miR-1256 mimic, ${ }^{*} \mathrm{P}<0.05$. (D) Quantitative analysis of TCTN1 expression in NSCLC and adjacent normal tissues ( $\mathrm{n}=30$, $\left.{ }^{* * *} \mathrm{P}<0.01\right)$. (E) TCTN1 protein expression in A549 cells and SK-MES-1 cells after transfected with control, miR-1256 mimic/inhibitor was detected by western blot analysis. (F and G) TCTN1 mRNA expression in A549 cells and SK-MES-1 cells after transfected with control, miR-1256 mimic/inhibitor was detected by qRT-PCR, ${ }^{\text {P }<0.05 ; ~}{ }^{\#} \mathrm{P}<0.05$. TCTN1, tectonic family member 1; qRT-PCR, quantitative real-time PCR; 3'-UTR, 3'-untranslated region.

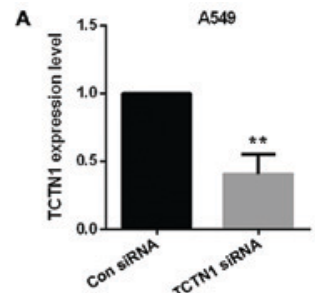

C

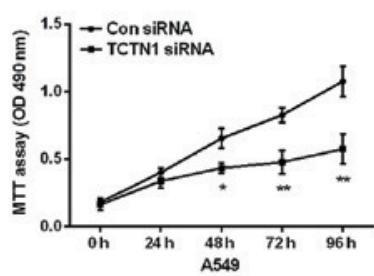

B

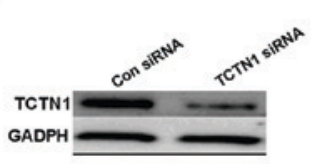

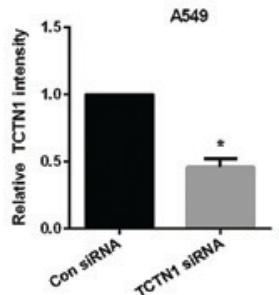

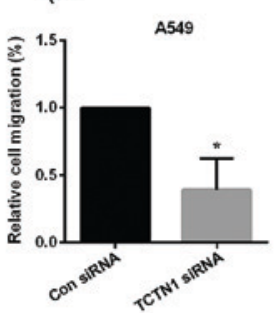

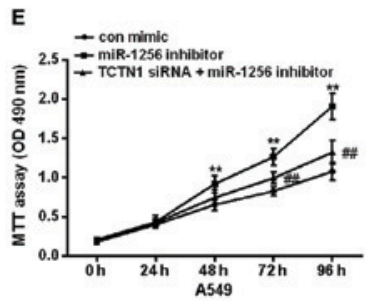

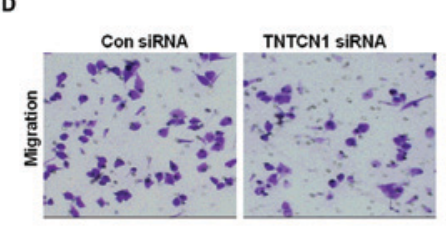

$\mathbf{F}$
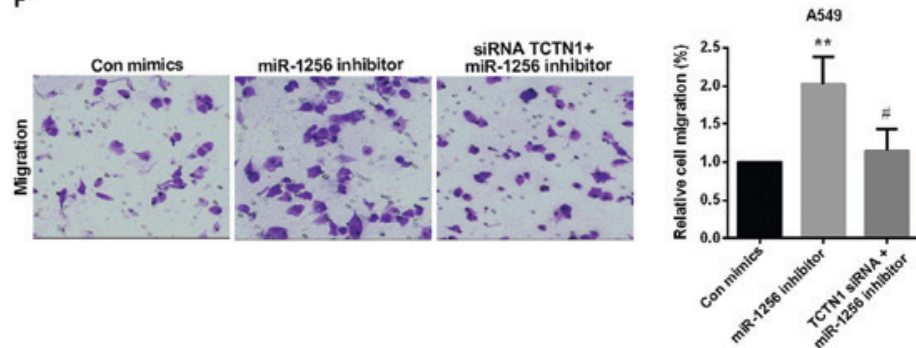

Figure 4. Knockdown of TCTN1 inhibits NSCLC cell proliferation and migration and TCTN1 reversed partial function of miR-1256. (A and B) The TCTN1 expression level in NSCLC cells after transfected with TCTN1 siRNA was detected by qRT-PCR and western blot analysis, ${ }^{*} \mathrm{P}<0.05,{ }^{* * *} \mathrm{P}<0.01$. (C and D) MTT and transwell assay, A549 cell proliferation and migration after knockdown of TCTN1, ${ }^{*} \mathrm{P}<0.05,{ }^{* * *} \mathrm{P}<0.01$. (E and F) Treatment with both miR-1256 inhibitor and siRNA TCTN1 reversed the promotion of A549 cell proliferative and migratory capacities compared with treatment with miR-1256 inhibitor alone, ${ }^{* *} \mathrm{P}<0.01 ;{ }^{*} \mathrm{P}<0.05 ;{ }^{\# \#} \mathrm{P}<0.01$. TCTN1, tectonic family member 1; NSCLC, non-small cell lung cancer; qRT-PCR, quantitative real-time PCR; MTT, methyl thiazolyl tetrazolium assay. 
and progression of NSCLC. We also confirmed the inhibitory effect of miR-1256 in NSCLC cell proliferation and migration, overexpression of miR-1256 could reduce the proliferative and migratory cell numbers, whereas, miR-1256 inhibitor facilitated cell migration and proliferation. Furthermore, we first identified that miR-1256 directly targeted TCTN1 in regulating of the development of NSCLC using luciferase assay.

TCTN1 was proved to participate in various developmental processes as a novel oncogene and the expression of TCTN1 in multiple solid tumors was abnormal (27). TCTN1 was also involved in a variety of biological processes. Many studies have reported that knockdown of TCTN1 inhibited cell growth in different types of malignant tumor cells. TCTN1 participated in the growth of gastric cancer cells, prostate cancer cells, human glioblastoma, pancreatic cancer and medulloblastoma $(16,17,19,28,29)$. In the present study, we found that TCTN1 expression in NSCLC was increased compared with normal tissues, and inhibiting TCTN1 could suppress the proliferation and migration of NSCLC cells. The protein and mRNA expression showed that the expression of miR-1256 and TCTN1 was negatively correlated in NSCLC. Additionally, we found that TCTN1 could reverse partial function of miR-1256 in regulating NSCLC development.

Collectively, the above study proved that miR-1256 played an important role in regulating NSCLC cell proliferation and migration. Moreover, miR-1256 mimic inhibited NSCLC cell proliferation and migration via directly targeting TCTN1. Therefore, miR-1256 could be used as a therapeutic cell target for treating NSCLC patients.

\section{Acknowledgements}

Not applicable.

\section{Funding}

No funding was received.

\section{Availability of data and materials}

The datasets used and/or analyzed during the present study are available from the corresponding author on reasonable request.

\section{Authors' contributions}

JZ and XH contributed to the conception of the study. LW and WL contributed significantly to the data analysis and study preparation. ZM, WL and FL performed the data analyses and wrote the study. XW helped perform the analysis with constructive discussions. All authors have read and approved the final study.

\section{Ethics approval and consent to participate}

All this experiments were approved by the Ethics Committee of People's Hospital of Rizhao (Rizhao, China). Patients signed informed consent.

\section{Consent for publication}

Not applicable.

\section{Competing interests}

Authors declare that they have no competing interests.

\section{References}

1. Jemal A, Bray F, Center MM, Ferlay J, Ward E and Forman D: Global cancer statistics. CA Cancer J Clin 61: 69-90, 2011.

2. Siegel R, Naishadham D and Jemal A: Cancer statistics, 2012. CA Cancer J Clin 62: 10-29, 2012.

3. Lee CT, Risom T and Strauss WM: MicroRNAs in mammalian development. Birth Defects Res C Embryo Today 78: 129-139, 2006.

4. Bueno MJ, Pérez de Castro I and Malumbres M: Control of cell proliferation pathways by microRNAs. Cell Cycle 7: 3143-3148, 2008.

5. Jovanovic M and Hengartner MO: miRNAs and apoptosis: RNAs to die for. Oncogene 25: 6176-6187, 2006.

6. Cui H and Yang L: Analysis of microRNA expression detected by microarray of the cerebral cortex after hypoxic-ischemic brain injury. J Craniofac Surg 24: 2147-2152, 2013.

7. Shi $C$ and Zhang Z: Screening of potentially crucial genes and regulatory factors involved in epithelial ovarian cancer using microarray analysis. Oncol Lett 14: 725-732, 2017.

8. Ma DH, Li BS, Liu JJ, Xiao YF, Yong X, Wang SM, Wu YY, Zhu HB, Wang DX and Yang SM: miR-93-5p/IFNAR1 axis promotes gastric cancer metastasis through activating the STAT3 signaling pathway. Cancer Lett 408: 23-32, 2017.

9. Shen S, Huang K, Wu Y, Ma Y, Wang J, Qin F and Ma J: A miR-135b-TAZ positive feedback loop promotes epithelialmesenchymal transition (EMT) and tumorigenesis in osteosarcoma. Cancer Lett 407: 32-44, 2017.

10. Yuan Z, Baker K, Redman MW, Wang L, Adams SV, Yu M, Dickinson B, Makar K, Ulrich N, Böhm J, et al: Dynamic plasma microRNAs are biomarkers for prognosis and early detection of recurrence in colorectal cancer. Br J Cancer 117: 1202-1210, 2017.

11. PurfieldDC,Bradley DG,Kearney JFand Berry DP: Genome-wide association study for calving traits in Holstein-Friesian dairy cattle. Animal 8: 224-35, 2014.

12. Li Y, Kong D, Ahmad A, Bao B, Dyson G and Sarkar FH: Epigenetic deregulation of miR-29a and miR-1256 by isoflavone contributes to the inhibition of prostate cancer cell growth and invasion. Epigenetics 7: 940-949, 2012.

13. Krol J, Loedige I and Filipowicz W: The widespread regulation of microRNA biogenesis, function and decay. Nat Rev Genet 11: 597-610, 2010.

14. Calin GA and Croce CM: MicroRNA signatures in human cancers. Nat Rev Cancer 6: 857-866, 2006.

15. Clark HF, Gurney AL, Abaya E, Baker K, Baldwin D, Brush J, Chen J, Chow B, Chui C, Crowley C, et al: The secreted protein discovery initiative (SPDI), a large-scale effort to identify novel human secreted and transmembrane proteins: a bioinformatics assessment. Genome Res 13: 2265-2270, 2003.

16. Wang Z, Gao Y, Liu Y, Chen J, Wang J, Gan S, Xu D and Cui X: Tectonic-1 contributes to the growth and migration of prostate cancer cells in vitro. Int J Mol Med 36: 931-938, 2015.

17. Wang X, Yu Q, Zhang Y, Ling Z and Yu P: Tectonic 1 accelerates gastric cancer cell proliferation and cell cycle progression in vitro. Mol Med Rep 12: 5897-5902, 2015.

18. Dai X, Dong M, Yu H, Xie Y, Yu Y, Cao Y, Kong Z, Zhou B, $\mathrm{Xu}$ Y, Yang T, et al: Knockdown of TCTN1 strongly decreases growth of human colon cancer cells. Med Sci Monit 26: 452-461, 2017.

19. Meng D, Chen Y, Zhao Y, Wang J, Yun D, Yang S, Chen J, Chen H and Lu D: Expression and prognostic significance of TCTN1 in human glioblastoma. J Transl Med 12: 288, 2014.

20. Livak KJ and Schmittgen TD: Analysis of relative gene expression data using real-time quantitative PCR and the 2(-Delta Delta C(T)) Method. Methods 25: 402-408 (2001).

21. Del Vescovo V, Grasso M, Barbareschi M and Denti MA: MicroRNAs as lung cancer biomarkers. World J Clin Oncol 5: 604-620, 2014. 
22. Esquela-Kerscher A and Slack FJ: Oncomirs - microRNAs with a role in cancer. Nat Rev Cancer 6: 259-269, 2006.

23. Yang J, Lan H, Huang X, Liu B and Tong Y: MicroRNA-126 inhibits tumor cell growth and its expression level correlates with poor survival in non-small cell lung cancer patients. PloS one 7: e42978, 2012.

24. Hayashita Y, Osada H, Tatematsu Y, Yamada H, Yanagisawa K, Tomida S, Yatabe Y, Kawahara K, Sekido Y and Takahashi T: A polycistronic microRNA cluster, miR-17-92, is overexpressed in human lung cancers and enhances cell proliferation. Cancer Res 65: 9628-9632, 2005.

25. Zhang JG, Wang JJ, Zhao F, Liu Q, Jiang $\mathrm{K}$ and Yang GH: MicroRNA-21 (miR-21) represses tumor suppressor PTEN and promotes growth and invasion in non-small cell lung cancer (NSCLC). Clin Chim Acta 411: 846-852, 2010

26. Liu X, Sempere LF, Ouyang H, Memoli VA, Andrew AS, Luo Y, Demidenko E, Korc M, Shi W and Preis M: MicroRNA-31 functions as an oncogenic microRNA in mouse and human lung cancer cells by repressing specific tumor suppressors. J Clin Invest 120: 1298-309, 2010
27. Reiter JF and Skarnes WC: Tectonic, a novel regulator of the Hedgehog pathway required for both activation and inhibition. Genes Dev 20: 22-27, 2006.

28. Zhao S, Chen X, Wan M, Jiang X, Li C, Cui Y and Kang P: Tectonic 1 is a key regulator of cell proliferation in pancreatic cancer. Cancer Biother Radiopharm 31: 7-13, 2016.

29. Jing J, Wang C, Liang Q, Zhao Y, Zhao Q, Wang S and Ma J: Lentivirus-mediated knockdown of tectonic family member 1 inhibits medulloblastoma cell proliferation. Int J Clin Exp Med 8: 13127-13135, 2015.

This work is licensed under a Creative Commons Attribution-NonCommercial-NoDerivatives 4.0 International (CC BY-NC-ND 4.0) License. 